\title{
Scurvy due to restrictive diet in a child with autism spectrum disorder. Case report
}

\author{
Melina J. Saavedra M.D. ${ }^{a}$, Julieta Aziz M.D. ${ }^{a}$ and Nicolás Cacchiarelli San Román M.D. ${ }^{a}$
}

\begin{abstract}
Scurvy is a very uncommon entity in pediatric population. Some case reports have been published in infants fed with boiled milk, in patients with iron overload secondary to hematological diseases and in children with food restrictions secondary to neurodevelopmental disorders.

We report a case of a 4-year-old male patient with a diagnosis of autism spectrum disorder, which developed scurvy secondary to a long-term selective eating habit without fruit or vegetable intake.

The objective of this case report is to announce the pediatrician and other professionals dedicated to primary health care about scurvy as a potential consequence of restrictive diets in children with autism spectrum disorders as well as the importance of dietary history in sick patients to prevent associated micronutrient deficiencies.

Key words: scurvy, ascorbic acid, autism spectrum disorder, Feeding and Eating Disorders, Nutritional Deficiency.
\end{abstract}

http:/ / dx.doi.org/10.5546/ aap.2018.eng.e684

To cite: Saavedra MJ, Aziz J, Cacchiarelli San Román N. Scurvy due to restrictive diet in a child with autism spectrum disorder. Case report. Arch Argent Pediatr 2018;116(5):e684-e687.

\section{INTRODUCTION}

Scurvy is a disease caused by the nutritional deficiency of ascorbic acid (Vitamin C). Humans, unlike other living beings, cannot synthesize it. Consequently, they depend strictly on their exogenous contribution. ${ }^{1}$

a. Department of Pediatrics. Division of Clinical Pediatrics. Hospital Italiano de Buenos Aires. Ciudad de Buenos Aires.

\section{E-mail address:}

Melina J. Saavedra M.D.: melina.saavedra@hiba.org.ar

Funding: None.

Conflict of interest: None.

Received: 1-13-2018

Accepted: 4-18-2018
Currently, scurvy is a rare entity in the pediatric population. ${ }^{2}$ There are few reports in the literature describing the development of the disease in infants fed with boiled milk, as well as in patients with iron overload secondary to conditions such as sickle cell disease, thalassemia and bone marrow transplant and also in children with dietary restrictions secondary to psychiatric or neurodevelopmental disorders.-11

\section{CASE REPORT}

We report a case of a 4-year-old male patient with a diagnosis of autism spectrum disorder. He was examined in our institution due to hip pain and refusal to walk, associated with petechiae and bruising of the lower limbs.

She has been diagnosed with autism spectrum disorder since age 27 months and she has a family history contributory with a cousin with the same diagnosis.

The symptoms began one month prior to admission with gait claudication without another symptom. The physical examination revealed limitation to flexion-extension and rotation of both hips. An ultrasound was performed and was compatible with transient synovitis of the right hip. The patient looked irritable and developed contralateral hip pain. The joints seemed normal. X-Rays of both hips were normal and the ultrasound showed bilateral synovial thickening. The clinical manifestations were assumed as bilateral transient synovitis of the hip, so we reinforced the treatment with nonsteroidal anti-inflammatory drugs (NSAID).

The child persisted with the symptoms despite the treatment indicated and was referred to Rheumatology for further evaluation. The physical examination remained the same. Some laboratory tests were requested, which showed microcytic and hypochromic anemia (hemoglobin $10 \mathrm{mg} / \mathrm{dl}$ ), slightly increased erythrosedimentation (ESR) $(33 \mathrm{~mm} / \mathrm{h})$ whereas white blood cells, platelets and coagulogram were within normal limits.

The patient progressed with inability to walk and increased hip pain. He could not remain sitting or lying down, he just could calm down 
in his mother's arms. Because of worsening of symptoms, we decided to admit him for further evaluation in our service. Higher irritability was observed, with frog position of lower limbs (hips and knees in flexion and abduction), absence of active mobility and limitation to passive mobilization secondary to bilateral hip, knee, shoulder and right elbow pain. Diffuse petechiae were found on the lower limbs, as well as spontaneous bruising on the knees. (Figure 1) The laboratory tests were repeated, with persistent mild anemia and slightly elevated ESR.

FIGURE 1. Skin hemorrhagic lesions
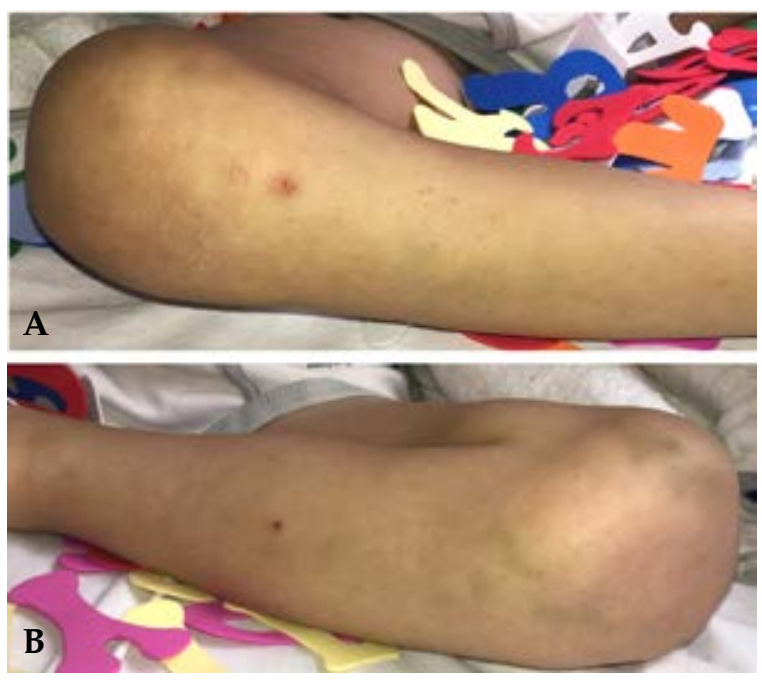

A y B. Diffuse petechiae in both lower limbs associated with ecchymosis.
Clinical manifestations persisted besides intravenously NSAID. Those symptoms raised concern about possibles rheumatological or oncohematological diagnosis. Therefore, a total body bone scan was performed, which was nonconclusive.

During the hospitalization and after an exhaustive anamnesis it was revealed that the child had selective feeding habit, due to the rejection of a wide variety of foods offered during meals. The restrictive habit began at age 18 months with consumption preferably of wheat semolina and dairy snacks, without intake of fruits or vegetables.

The patient weight was at the 25th percentile and his height at the 90th percentile. The weight for height $Z$ score was between -2 and -3 standard deviations, based on WHO`s standards. Scurvy was suspected as a possible diagnosis based on food restriction habits associated with the symptoms.

$X$-Rays of long bones were requested and findings compatible with scurvy were observed. (Figure 2)

Levels of Vitamin C in blood were measured and without waiting for results he started treatment with $300 \mathrm{mg}$ per day of ascorbic acid. Subsequently, the result of the test was below the normal limit $(0,5 \mathrm{mg} / \mathrm{dl}$, normal range between $0,6 \mathrm{mg} / \mathrm{dl}-2 \mathrm{mg} / \mathrm{dl})$.

The patient was discharged after pain improvement, with gait recovery after 5 days of treatment.

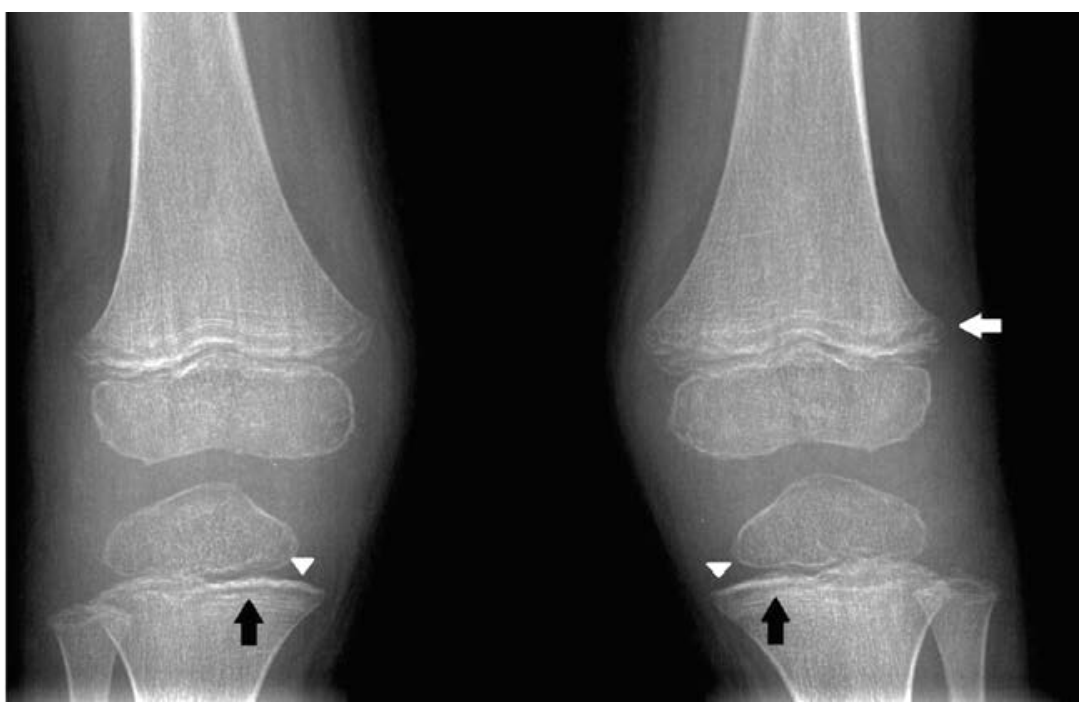

Anteroposterior radiograph of both knees shows metaphyseal irregularities with spurring (white arrow), a white line of Flankl in the zone of provisional calcification (white arrowheads) with metaphyseal lucent bands (Scurvy line or Trummerfeld zone) (black arrows). 
The child started nutritional follow-up with the aim of achieving a balanced diet suited to the patient's tastes. Other micronutrients, such as calcium and proteins, were found to be within normal values. Currently, he continues asymptomatic in follow-up with a pediatrician with a supply of ascorbic acid $100 \mathrm{mg}$ per day.

\section{DISCUSSION}

Scurvy is an ancient disease that has been recorded since $1500 \mathrm{BC}$. The treatment was described in 1753 by James Lind, an English naval doctor, when he observed that sailors with diagnosis of scurvy improved their symptoms 6 days after starting citrus ingestion. ${ }^{11,12}$

Autism spectrum disorder is a developmental condition characterized by a deficit in social interaction and restricted communication and interests. ${ }^{13,14}$ It has been reported that between $46 \%$ and $89 \%$ of these patients have feeding problems characterized by a selective diet with preference for carbohydrates, snacks and processed foods while rejecting fruits and vegetables intake. ${ }^{10}$ This behavior can lead to a deficit of macro and micronutrients. Most of these cases were published as reports or series of cases. ${ }^{3-9}$ In Sharp et al. ${ }^{10}$ meta-analysis was described a nutritional deficit of calcium and proteins in children with autism spectrum disorder compared with healthy peers; in addition to a lower intake of vitamins A, C, D and $\mathrm{E}$, nevertheless the difference for these nutrients was not statistically significant. ${ }^{10}$ Frequently these patients have adequate energy consumption, reaching the daily caloric requirement, so they achieve child growth standards. Consequently, the nutritional deficit is masked until the onset of symptoms. ${ }^{10}$

Ascorbic acid is an essential cofactor for the hydroxylation in collagen synthesis and for the hydroxylation of the neurotransmitter dopamine to noradrenaline. Many dermatological and musculoskeletal signs and symptoms are due to defects in collagen and chondroitin sulphate synthesis relate to vitamin $C$ deficiency. ${ }^{6}$

As presented in our patient, scurvy frequently manifests with hyperkeratosis and hemorrhagic skin lesions, mostly as ecchymoses and petechiae with perifollicular distribution. Likewise, gingival hypertrophy associated with bleeding and areas of necrotic gingiva were reported. ${ }^{3-10}$ Musculoskeletal manifestations were the reason for the child's complaining in our case, the disease can progress with generalized pain and edema, mostly in lower limbs determining the antalgic frog position, limping and/or refusal to walk. $^{3-10}$

It is described that signs and symptoms of scurvy develop after 1 to 3 months of inadequate intake of vitamin C (less than $10 \mathrm{mg} /$ day). ${ }^{11}$

The diagnosis is based on clinical manifestations, however some diagnostic tests are helpful. Mild anemia and slightly increased acute phase reactants (erythrocyte sedimentation and C-reactive protein) is usually manifested. Our patient showed laboratory tests and X-Rays compatible with the diagnosis and a nonconclusive body bone scan.

Currently, given the scurvy low suspicion, patients undergo multiple diagnostic tests in order to rule out onco-hematological and rheumatological diseases..$^{2-5,7,8}$

Clinical manifestations were improved by treatment with acid ascorbic and scurvy was confirmed. After starting the treatment, the enhancement of general symptoms is described in the first 24 hours, while the decrease in pain in 2 or 3 days and the musculoskeletal symptoms improvement in a few weeks. ${ }^{15}$ Our patient started treatment with acid ascorbic $300 \mathrm{mg}$ per day, consequently the analgesic requirement decreased and after 5 days he was able to stand and walk.

Decreased levels of ascorbic acid can support the diagnosis. In the literature, patients with scurvy who underwent dosing had values below $0.2 \mathrm{mg} / \mathrm{dl}$, which are lower than those found in our patient $(0.5 \mathrm{mg} / \mathrm{dl})$, however the signs and symptoms developed and the prompt response to treatment with vitamin $C$ confirmed the diagnostic suspicion.

\section{CONCLUSIONS}

Food selectivity by texture, type or presentation is one of the challenges faced by children with autism spectrum disorders.

A nutritional features assessment should be part of the pediatric check up, specially in this population and in sick patients, in order to avoid the development of micronutrient deficiency diseases.

Besides, pediatricians should consider the scurvy diagnosis in these patients, especially when they develop limping or refuse to walk and/or show petechiae, ecchymosis or gingival hypertrophy. Although a variety of initial tests are usually required to rule out other causes, proper suspicion of this disease would help to avoid unnecessary diagnostic tests and early 
treatment can help to revert the symptoms and prevent complications.

\section{Acknowledgements}

To the medical doctors Lucía Pérez and Celeste Puga for the review of the manuscript and advice.

\section{REFERENCES}

1. Levine M. New concepts in the biology and biochemistry of ascorbic acid. N Engl J Med 1986; 314(14):842-902.

2. Weinstein M, Babyn P, Zlotkin S. An orange a day keeps the doctor away: scurvy in the year 2000. Pediatrics 2001; 108(3):e55.

3. Niwa T, Aida N, Tanaka Y, et al. Scurvy in a child with autism: Magnetic Resonance Imaging and pathological findings. J Pediatr Hematol Oncol 2012; 34(6):484-7.

4. Golriz F, Donnelly LF, Devaraj S, et al. Modern American Scurvy - Experience With Vitamin C Deficiency at a Large Children's Hospital. Pediatr Radiol 2016; 47(2):214-20.

5. Ma NS, Thompson C, Weston S. Brief Report: Scurvy as a Manifestation of Food Selectivity in Children with Autism. J Autism Dev Disord 2015; 46(4):1464-70.

6. Duggan CP, Westra SJ, Rosenberg AE. Case records of the Massachusetts General Hospital. Case 23-2007. A 9-yearold boy with bone pain, rash, and gingival hypertrophy. N Engl J Med 2007; 357(4):392-400.
7. Gongidi P, Johnson C, Dinan D. Scurvy in an autistic child: MRI findings. Pediatr Radiol 2013; 43(10):1396-9.

8. Kitcharoensakkul M, Schulz CG, Kassel R, et al. Scurvy revealed by difficulty walking: Three cases in young children. J Clin Rheumatol 2014; 20(4):224-8.

9. Sharp WG, Berry RC, McCracken C, et al. Feeding problems and nutrient intake in children with autism spectrum disorders: A meta-analysis and comprehensive review of the literature. J Autism Dev Disord 2013; 43(9):2159-73.

10. Larralde $M$, Santos Muñoz A, Boggio $P$, et al. Scurvy in a 10-month-old boy. Int J Dermatol 2007; 46(2):194-8.

11. Hodges RE, Hood J, Canham JE, et al. Clinical manifestations of ascorbic acid deficiency in man. Am J Clin Nutr 1971; 24(4):432-43.

12. Carpenter KJ. A short history of nutritional science: Part 1 (1785 - 1885). J Nutr 2003; 133(3):638-45.

13. Developmental Disabilities Monitoring Network Surveillance Year 2010 Principal Investigators; Centers for Disease Control and Prevention (CDC). Prevalence of autism spectrum disorder among children aged 8 years - Autism and developmental disabilities monitoring network, 11 sites, United States, 2010. MMWR Surveill Summ 2014; 63(2):1-21.

14. American Psychiatric Association. Diagnostic and statistical manual of mental disorders. 4th ed. Washington, DC: American Psychiatric Association; 2000.

15. HirschmannJV, Raugi GJ. Adult scurvy. J Am Acad Dermatol 1999; 41(16):895-906. 